\title{
General Psychiatry PAID study design on the role of PKC activation in immune/inflammation- related depression: a randomised placebo-controlled trial protocol
}

Xiaoyun Guo (D) , ${ }^{1}$ Ruizhi Mao, ${ }^{1}$ Lvchun Cui, ${ }^{1}$ Fan Wang, ${ }^{1}$ Rubai Zhou, ${ }^{1}$ Yun Wang, ${ }^{1}$ Jia Huang, ${ }^{1}$ Yuncheng Zhu, ${ }^{1}$ Yamin Yao, ${ }^{1}$ Guoqing Zhao, ${ }^{2}$ Zezhi Li, ${ }^{3}$ Jun Chen (D) , ${ }^{4}$ Jinhui Wang, ${ }^{5}$ Yiru Fang (D) ${ }^{4,6,7}$

To cite: Guo X, Mao R, Cui L, et al. PAID study design on the role of PKC activation in immune/ inflammation-related depression: a randomised placebo-controlled trial protocol. General Psychiatry 2021;34:e100440. doi:10.1136/ gpsych-2020-100440

$\mathrm{XG}$ and $\mathrm{RM}$ contributed equally.

$\mathrm{XG}$ and $\mathrm{RM}$ are joint first authors.

Received 05 November 2020 Revised 18 February 2021 Accepted 02 March 2021
Check for updates

(C) Author(s) (or their employer(s)) 2021. Re-use permitted under CC BY-NC. No commercial re-use. See rights and permissions. Published by BMJ.

For numbered affiliations see end of article.

Correspondence to Dr Yiru Fang; yirufang@aliyun.com

\section{ABSTRACT}

Background Inflammation that is mediated by microglia activation plays an important role in the pathogenesis of depression. Microglia activation can lead to an increase in the levels of proinflammatory cytokines, including TNF- $\alpha$, which leads to neuronal apoptosis in the specific neural circuits of some brain regions, abnormal cognition and treatment-resistant depression (TRD). Protein kinase C (PKC) is a key regulator of the microglia activation process. We assume that the abnormality in PKC might result in abnormal microglia activation, neuronal apoptosis, significant changes in emotional and cognitive neural circuits, and TRD. In the current study, we plan to target at the PKC signal pathway to improve the TRD treatment outcome.

Methods and analysis This is a 12-week, ongoing, randomised, placebo-controlled trial. Patients with TRD ( $\mathrm{N}=180)$ were recruited from Shanghai Mental Health Center, Shanghai Jiao Tong University. Healthy control volunteers $(\mathrm{N}=60)$ were recruited by advertisement. Patients with TRD were randomly assigned to 'escitalopram+golimumab (TNF- $\alpha$ inhibitor)', 'escitalopram+calcium tablet+vitamin D (PKC activator)' or 'escitalopram+placebo' groups. We define the primary outcome as changes in the 17-item Hamilton Depression Rating Scale (HAMD-17). The secondary outcome is defined as changes in anti-inflammatory effects, cognitive function and quality of life.

Discussion This study might be the first randomised, placebo-controlled trial to target at the PKC signal pathway in patients with TRD. Our study might help to propose individualised treatment strategies for depression.

Trial registration number The trial protocol is registered with ClinicalTrials.gov under protocol ID 81930033 and ClinicalTrials.gov ID NCT04156425.

\section{BACKGROUND}

\section{Background and rationale}

Depression is a severe mental disease characterised by significant and persistent low mood. Depression has a high prevalence rate, high recurrence rate, high disability rate, high suicide rate and high disease burden.
At present, the exact pathogenesis of depression is not well understood. Previous studies have shown that neurobiochemical mechanisms contribute to the pathogenesis of depression. ${ }^{2}$ However, almost $50 \%$ of patients with depression do not respond satisfactorily to the antidepressants developed primarily according to the aforementioned neurotransmitter/receptor theory. Treatment-resistant depression (TRD) is defined as an insufficient response (response rate $<50 \%$ ) to two antidepressants with different mechanisms when given for at least 6 weeks at an adequate dose. Although many novel treatment methods have appeared in the past several years, ${ }^{3}$ the treatment of the TRD remains one of the great challenges to psychiatrists.

The proinflammatory cytokines, particularly tumour necrosis factor-alpha (TNF- $\alpha$ ), play an important role in the therapeutic outcome of antidepressant treatment. ${ }^{4} \mathrm{~A}$ subset of patients with depression showed elevated plasma levels of TNF- $\alpha .{ }^{5}$ The baseline levels of proinflammatory cytokines might be a predictive parameter for antidepressant treatment prognosis. ${ }^{4}$ Anti-inflammatory substances, such as monoclonal antibodies and anti-inflammatory cytokines, have potentially effective antidepressant effects, as evidenced by their alleviation of depressivelike behaviour in animal models and their antidepressant effect in clinical trials. ${ }^{6}$

Microglia are the key immune cells in the central nervous system (CNS). After stress, the resting microglia could be activated into two subtypes, the pro-inflammatory M1 phenotype and the anti-inflammatory M2 phenotype. ${ }^{7}$ M1 microglia can synthesise and release several proinflammatory cytokines, while M2 microglia can synthesise and release anti-inflammatory cytokines. ${ }^{89}$ 


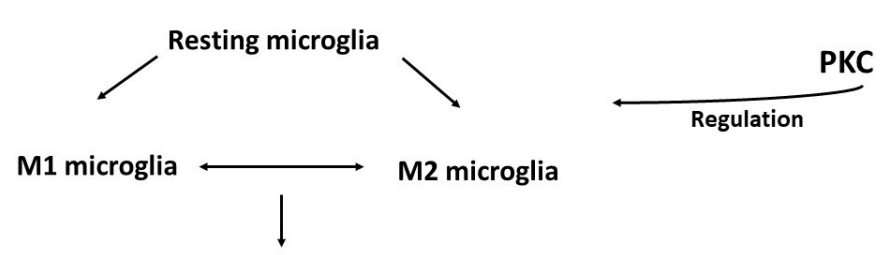

Neurodegeneration and apoptosis

Cognitive dysfunction

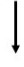

TRD

Figure 1 Protein kinase $\mathrm{C}(\mathrm{PKC})$ signalling pathway during the immune-inflammatory pathogenesis of TRD. Resting microglia could be activated into two subtypes: pro-inflammatory M1 microglia and anti-inflammatory M2 microglia. Maintaining a balanced state for the M1/ M2 microglia is important for maintaining normal immunity in the CNS. Abnormal communication between microglia and neurons might lead to neuronal apoptosis, cognitive dysfunction and TRD. CNS, central nervous system; TRD, treatment-resistant depression.

A balanced state of M1/M2 microglia is important for maintaining normal immunity and the physiological state of the CNS. ${ }^{10}$ Excessive M1 microglia activation might contribute to the aetiology of depression, as evidenced by rodents ${ }^{9}$ in a clinical study. ${ }^{11}$ Since microglia and neurons can send signals to one another, the abnormal communication between them might lead to neuronal apoptosis, ${ }^{12}$ cognitive dysfunction ${ }^{13}$ and poor treatment response. ${ }^{14}$ Protein kinases might play an important role in regulating microglia activation, among which PKC might be the most prominent player by phosphorylating the substrate protein. ${ }^{15} 16$ The downregulation of PKC mediates the LPS-induced inflammatory response and the regulation towards M1 phenotype activation. ${ }^{16}$ Meanwhile, PKC activation induces a significant neuroprotective effect, as evidenced by enhanced BDNF activation, and promotes neurogenesis in the hippocampal dentate gyrus and the subventricular zone ${ }^{10}$ (figure 1). Interestingly, our previous research showed that the mRNA expression of the PKC $\beta 1$ isoform (PRCKB1) in peripheral blood mononuclear cells (PBMCs) is significantly downregulated in depressed patients. ${ }^{17}$ Hence, our hypothesis is as follows: drugs that target the PKC signal pathway may alleviate the symptoms of depression, especially in TRD.

So far, some RCT studies that have targeted TNF- $\alpha$ antagonists (eg, infliximab) failed to show the generalised efficacy in treatment-resistant depression; however, the results indicated improvement of depressive symptoms in patients with high baseline inflammatory biomarkers. ${ }^{6}$ A well-designed RCT study is needed to explore the therapeutic outcome in TRD, targeting the PKC and related immune-inflammatory signal pathway. We choose vitamin D as a PKC signal pathway activator to treat patients with TRD. That is because vitamin $\mathrm{D}$ helps the body absorb calcium. While the activation of PKC requires sufficient $\mathrm{Ca}^{2+}$ concentrations, ${ }^{18}$ drugs that induce increased levels of calcium may act as activator of PKC. In the current study, we will carry out a randomised placebo-controlled trial to study the treatment outcome for vitamin D (PKC activator) and golimumab (TNF- $\alpha$ inhibitor $)^{19}$ that target the PKC activation and its related immune-inflammatory signal pathway in patients with TRD (PAID study). Based on the current study, we hope to provide more evidence and shed new light on therapeutic approaches to TRD.

\section{Objectives}

\section{Primary objective}

The primary objective of this study is to investigate the antidepressant efficacy, measured by the 17-item Hamilton Depression Rating Scale (HAMD-17) score, of the 12-week treatment of antidepressant (escitalopram) plus anti-inflammatory medication (golimumab) or PKC activator (calcium tablet+vitamin $\mathrm{D}$ ). We hypothesis that a higher efficacy and larger reduction in symptoms of depression will be observed after 12 weeks when treatment is combined (antidepressant+anti-inflammatory or antidepressant+PKC activator) compared to treatment with an antidepressant alone.

\section{Secondary objectives}

1. To investigate the anti-inflammatory effects of combined treatment of TRD with a PKC activator and antiinflammatory drugs.

2. To investigate the effects of a PKC activator and antiinflammatory drug on cognition.

3. To investigate the effects of a PKC activator and antiinflammatory drug on quality of life.

We hypothesise that combined treatment of TRD with a PKC activator and anti-inflammatory drug will result in anti-inflammatory effects, and improve the cognitive function and quality of life in patients with TRD.

\section{Trial design}

We adopted a randomised allocation design to test placebo-controlled antidepressant augmentation. TRD patients who had not previously received escitalopram or who had received escitalopram doses of less than $20 \mathrm{mg} / \mathrm{d}$ were randomised (1:1:1) into one of the following three groups: 'escitalopram+golimumab', 'escitalopram+calcium tablet+vitamin D' or 'escitalopram+placebo'. The total study duration is 12 weeks (figure 2).

\section{METHODS AND DESIGN FOR THE STUDY \\ Setting}

Treatment is given at the Shanghai Mental Health Center outpatient or inpatient units. Functional MRI (fMRI) scanning and PET scanning is performed at the Imaging Department at Shanghai Mental Health Center and the Imaging Department at Shanghai Renji Hospital. 


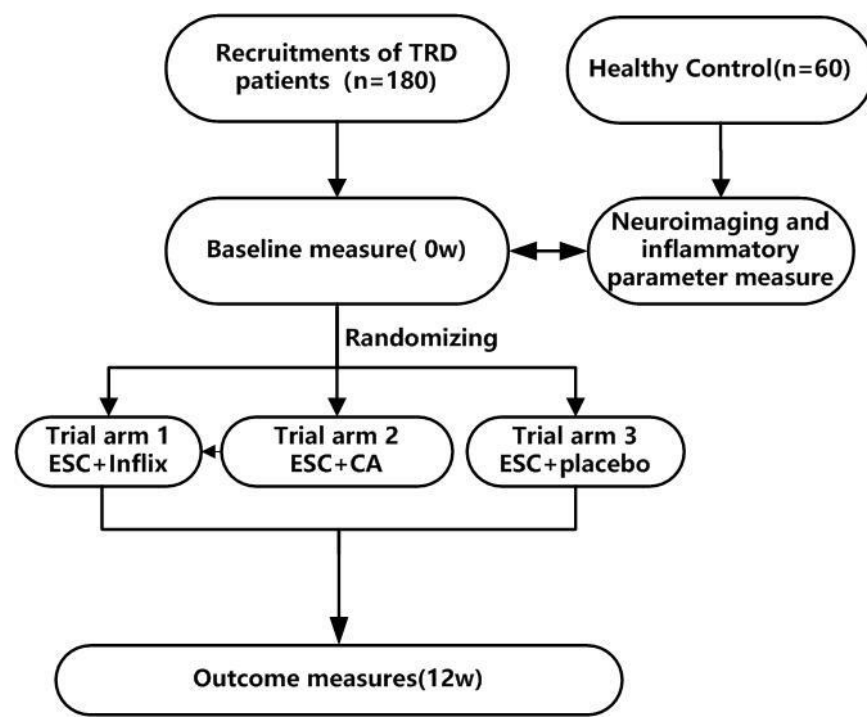

Figure 2 Trial flowchart. We plan to recruit 180 patients with TRD and 60 healthy controls. For the patients who do not receive escitalopram or who received escitalopram at less than $20 \mathrm{mg} / \mathrm{d}$, the patients will be randomised (1:1:1) into one of the following three groups: 'escitalopram+golimumab', 'escitalopram+calcium tablet $500 \mathrm{mg} / \mathrm{d}$ +vitamin D $800 \mathrm{lU} / \mathrm{d}$ ' or 'escitalopram+placebo' groups. Primary and secondary study parameters will be accessed at baseline (week 0 ) and the 12th week. CA, calcium; ESC, escitalopram; Inflix, infliximab; TRD, treatment-resistant depression.

\section{Eligibility criteria}

Inclusion criteria for TRD

1. Diagnosed with major depressive disorder (MDD) according to the Diagnostic and Statistical Manual of Mental Disorders, Fifth Edition (DSM-5);

2. Insufficient response (response rate <50\%) to two antidepressants with different mechanisms when given for at least 6 weeks at an adequate dose (eg, clomipramine $\geq 150 \mathrm{mg} / \mathrm{d}$, fluoxetine $\geq 20 \mathrm{mg} / \mathrm{d}$ ) during the current episode;

3. A 17-item Hamilton Depression Rating Scale (HAMD17) score $\geq 17$ no more than 7 days prior to randomisation. Cognitive factors (including a sense of guilt, suicidal thoughts, agitation, depersonalisation, the disintegration of reality, paranoid symptoms, and obsessive and compulsive symptoms) score $\geq 6$;

4. Between 18 and 65 years of age;

5. Education: finished middle school;

6. Ethnicity: Han Chinese;

7. Adequate auditory and visual ability to complete the necessary checks;

8. Compliance with treatment in the clinical trial.

\section{Exclusion criteria for TRD}

1. Severe liver and kidney diseases, active endocrine diseases or clinical symptoms. Severe cardiovascular disease, respiratory system disease, haematologic diseases and cancer;

2. Serious suicide attempts;

3. Pregnancy or lactation;
4. Receiving modified electroconvulsive therapy (MECT) in the past 1 month;

5. Known current psychosis as determined by the DSM5 or a history of a non-mood psychotic disorder;

6. Participation in another clinical trial concurrently or no more than 1 month prior to randomisation;

7. Previously had a severe allergic reaction or immune system disease;

8. Using an anti-inflammatory drug or immunosuppressive agent;

9. HAMD-17 item 3 (suicide) score $\geq 3$;

10. Patients who had received a sufficient dose $(20 \mathrm{mg} / \mathrm{d})$ and sufficient course (more than 4-6 weeks) of escitalopram.

\section{Inclusion criteria for healthy controls}

1. Healthy participants of matched age, sex and education with the TRD group;

2. A willingness to adhere to all rules necessary for the study;

3. Signed informed consent.

\section{Exclusion criteria for healthy controls}

1. Severe physical diseases, or a history of traumatic brain injury;

2. Serious allergic diseases of the immune system;

3. Use of anti-inflammatory drugs or immunomodulatory drugs no more than 1 month prior to randomisation;

4. Pregnancy or lactation.

\section{Interventions}

There are three treatment types: 'escitalopram+golimumab', 'escitalopram+calcium tablet $500 \mathrm{mg} / \mathrm{d}$ +vitamin D $800 \mathrm{IU} / \mathrm{d}$ ' and 'escitalopram+placebo'. Participants receive their usual medical care while participating in this study. Antidepressants are restricted to the selective serotonin reuptake inhibitor escitalopram $(20 \mathrm{mg} / \mathrm{d})$.

Participants can leave the study at any time for any of the following reasons:

1. If participants do not meet the inclusion criteria;

2. If participants or their guardians decide to leave the study;

3. If participants develop serious adverse events related to the study medication;

4. If participants develop a serious physical disease whether or not it is associated with the study medication;

5. If participants stop taking their trial medication for three consecutive days, stop effective contraception or become pregnant;

6. If participants use other treatments (including MECT);

7. If other conditions occur, and the investigator decides to terminate the study;

8. Defaulting.

Two independent individuals in the nursing department count the pills and golimumab ampoules to check patient adherence. Collaboration with patient guardians also helps the patients adhere to the treatment. 
Benzodiazepines (BZDs) are allowed as part of the treatment if determined to be necessary by the treating physician. The use of BZDs is carefully recorded throughout the whole trial. The golimumab and calcium tablet treatment is in addition to ongoing antidepressant treatment. During the study, it is not permitted to change the antidepressants or use ECT treatment (see figure 1). Golimumab is administered at a subcutaneous dose of $50 \mathrm{mg}$ at the baseline and every month (baseline, 1 month and 2 months). Calcium tablets are administered at $500 \mathrm{mg} / \mathrm{d}$. Vitamin D is administered at $800 \mathrm{IU} / \mathrm{d}$.

\section{Outcomes}

Primary outcome: The primary outcome is defined as changes in depressive symptoms between baseline and 12 weeks after the treatment.

Secondary outcomes: The secondary outcome is defined as changes in cognitive function, local brain activity, biomarkers representing inflammatory mechanisms associated with PKC activation and microglia activation.

\section{Sample size}

The sample size was calculated based on the data from Bekhbat $e t a l^{6}$ and Khoraminya $e t a l .^{20}$ In Bekhbat $e t a l \mathrm{~s}^{6}$ study, the results showed that TNF- $\alpha$ antagonist infliximab reduced depressive symptoms in patients with TRD with an effect size of 0.368 . In Khoraminya $e t a l \mathrm{~s}^{20}$ study, the vitamin D combination therapy was superior to antidepressant fluoxetine alone in controlling depression with an effect size of 0.414 . We set $\alpha$ at 0.05 , effect size as 0.3 , number of measurement as 2 and the power at 0.80 in MANOVA: repeated measures, within-between interactions. We adopted G power software (V.3.1.9.4) to calculate the sample size. The result indicated that a minimum of 37 patients per group is needed (a total of 111 patients). Considering the 20\% drop-out rate, 133 patients are needed in our experiment. Finally, we planned to enrol 60 patients per group for a total of 180 included patients.

\section{Recruitment}

A well-trained group is involved in the participant enrolment process, including one senior psychiatrist, two nurses, two PhD students working full-time and 10 psychiatrists who refer patients for scientific research. We provide the participants and their guardians with a thorough explanation to ensure that they fully understand the study.

The Shanghai Mental Health Center is one of the top two largest mental health facilities in China, with approximately 600000 outpatients every year and 1300 beds. We are sure to finish the study schedule using the current protocol.

\section{Allocation}

Participants will be randomly assigned to either control or experimental group with a 1:1:1 allocation as per a computer-generated randomisation schedule stratified whether they had previously received a sufficient dose
(20 mg/d) and sufficient course (more than 4-6 weeks) of escitalopram.

The investigators are blinded to the treatment allocation until the data analysis is completed. A person who is not involved in any other part of the trial performs the randomisation procedure.

Two senior psychiatrists experienced with the MINI scale guarantee an accurate diagnosis. Moreover, we also checked the internal consistency for all psychiatrists involved in scale ratings with a kappa value of 0.81 . All patients who fulfil the inclusion criteria will be randomised. Randomisation will be requested by the psychiatrists who are responsible for recruitment and clinical interview. The randomisation number in an envelope will be sent to the psychiatrists of the patient. The psychiatrists will open the envelope, find the treatment information and give the treatment to the patient.

\section{Blinding}

Staff responsible for the recruitment and symptom evaluation are blind to the information about group allocation. A staff member outside the research group will feed data into the computer.

\section{Data collection methods}

Primary study parameter/endpoint

HAMD-17: The primary outcome criterion is the change in HAMD-17 score before and after 12 weeks of the treatment. A treatment response is defined as a $50 \%$ reduction in the HAMD-17 score at any point during the 12-week trial compared with the baseline.

\section{Secondary study parameters}

1. Mini International Neuropsychiatric Interview (MINI);

2. Clinical Global Impression Scale (CGIS);

3. Quick Inventory of Depressive Symptomatology (SelfReported) (QIDS-SR-16);

4. Sheehan Disability Scale (SDS);

5. Quality of Life (QOL);

6. Montreal Cognitive Assessment (MoCA);

7. WHO Disability Assessment Schedule (WHODAS-II);

8. Neuroimaging: Microglia activation can be accessed using PET. ${ }^{21}$ The present study aims to visualise microglia activation by examining translocator protein (TSPO) total distribution volume (VT) in PET in the prefrontal cortex, hippocampus, anterior cingulate cortex and superior parietal before and after treatment. Patients receive two PET scan sessions before the treatment starts and at week 12. Patients will also receive two fMRI scan sessions before the treatment starts and at week 12. A resting state functional fMRI scan is recorded. A DTI scan with two fMRI tasks is performed to assess the connectivity changes after the treatment. MRI images are acquired with the corresponding PET images.

9. Peripheral venous blood: Biomarkers representing inflammatory mechanisms associated with MDD are assessed in the venous blood mononuclear cells. (1) Routine tests: The routine tests include measurements 
Table 1 Data collection methods and clinical assessment time points

\begin{tabular}{|c|c|c|c|}
\hline Scale 1 & MINI & \multicolumn{2}{|c|}{$\begin{array}{l}\text { Psychiatric structured } \\
\text { diagnostic interview } \\
\text { instrument }\end{array}$} \\
\hline \multirow[t]{8}{*}{ Scale 2} & HAMD-17 & \multicolumn{2}{|c|}{$\begin{array}{l}\text { Self-rating scale for } \\
\text { depressive symptoms }\end{array}$} \\
\hline & CGIS & \multicolumn{2}{|c|}{ To access severity of disease } \\
\hline & QIDS-SR-16 & \multicolumn{2}{|c|}{$\begin{array}{l}\text { Self-reported inventory for } \\
\text { depressive symptoms }\end{array}$} \\
\hline & SDS & \multicolumn{2}{|c|}{ To access functioning } \\
\hline & QOL & \multicolumn{2}{|c|}{ To access functioning } \\
\hline & MoCA & \multicolumn{2}{|c|}{ To access cognition } \\
\hline & WHODAS-II & \multicolumn{2}{|c|}{ To access functioning } \\
\hline & $\begin{array}{l}\text { Scale } \\
\text { assessments }\end{array}$ & Neuroimaging & $\begin{array}{l}\text { Biomarkers in } \\
\text { peripheral venous } \\
\text { blood }\end{array}$ \\
\hline Baseline & $\begin{array}{l}\text { Scale 1, Scale } \\
2\end{array}$ & $\sqrt{ }$ & $\sqrt{ }$ \\
\hline Week 12 & Scale 2 & $\sqrt{ }$ & $\sqrt{ }$ \\
\hline
\end{tabular}

CGIS, Clinical Global Impression Scale; HAMD-17, 17-item Hamilton Depression Rating Scale; MINI, Mini International Neuropsychiatric Interview; MoCA, Montreal Cognitive Assessment; QIDS-SR-16, Quick Inventory of Depressive Symptomatology (Self-Reported); QOL, Quality of Life; SDS, Sheehan Disability Scale; WHO-DAS II, WHO Disability Assessment Schedule.

of the white blood count, $\mathrm{C}$ reactive protein (CRP), hepatic and renal function, glucose, serum vitamin $\mathrm{D}$, etc. (2) Specific tests: We collect the peripheral blood serum and store it at $-80^{\circ} \mathrm{C}$ after centrifugation. We examine the PKC and other inflammatory markers in serum using ELISA. We also collect the whole blood, extract the PBMC and evaluate markers of mononuclear activation using flow cytometry.

\section{Participant timeline}

All the time points for the physical examinations, scale assessments, medication use inventories and laboratory examinations are shown in table 1.

\section{Data management}

In the PAID study, all data will be entered electronically. All of the data management work will be done at a Core Coordinating Center at Shanghai Mental Health Center. A special database will be built to record all the data related to PAID study. A complete back-up of the primary database will be performed once a month.

A Data Monitoring Committee (DMC) has been established. The DMC is independent of the study organisers. We perform an interim analysis on the primary endpoint when $30 \%$ and $70 \%$ of patients have been randomised and have completed the 12-week follow-up. The interim analysis is performed by an independent statistician. The statistician will report independently to the DMC.

\section{Statistical methods}

We summarise the numerical data in normal distributions by using the means and SDs. We will present the numerical data in non-normal distributions with the median and IQR. The MANOVA test will be used to compare numerical data (eg, sociodemographic data, cognitive function data, biomarkers representing inflammatory mechanisms associated with PKC activation and microglia activation data) among groups if data were normally distributed. Non-parametric tests will be used if data were not normally distributed. Chi-square analyses will be used to compare count data (eg, response rate among groups). We will combine intention-to-treat analysis and per-protocol analysis, using a mixed-effects model for repeated measures to analyse change from baseline of HAMD scores, time and their interaction. We will also adopt pretreatment values (eg, age, gender, baseline HAMD, CRP and vitamin D level) as covariates to correct the possible differences. Quadratic terms will be used if the data exhibit a unique convex. We set $\alpha$ of 0.05 as significant.

\section{Harms}

We record the possible side effects for each participant. Our trained team reports these data throughout the study using the serious adverse event reporting system.

\section{ETHICS AND DISSEMINATION}

Trained research nurses will introduce the trial and information sheets to patients. Research nurses will get written consent from patients and their supervisors. All information sheets and consent forms have been translated into Chinese and English. There are separate information sheets and consent forms for the depression and healthy control group.

Additional serum and plasma samples will be obtained to be stored for future use. An informed consent will be obtained to specifically address the collection of these samples.

All study-related information will be stored at the study site. All participant information will be stored in locked file cabinets in areas with limited access. All laboratory specimens and data reports will be accessed by a coded ID number. All records that contain names or other personal identifiers will be stored separately from study records. All local databases will be accessed by password.

Principal investigators will be given access to the cleaned data sets. All data will be password protected. To ensure confidentiality, project team members will be blinded to the data.

Shanghai Jiao Tong University has the insurance to cover all non-negligent harm associated with the protocol. Incidences from negligence (eg, major protocol violations) will not be covered by study insurance.

The principal investigator (PI) will review all publications. The PI will be considered for lead author derived from this study. No later than 5 years after the ending of 
recruitment, we will deliver the whole deidentified data set to an appropriate data archive.

Any modifications to the protocol will require a formal amendment to the protocol.

\section{DISCUSSION}

Our project is the first randomised placebo-controlled trial to study the treatment outcome for TRD targeting the PKC and its related immune-inflammatory signal pathway.

The regulatory domain of the PKC acts as a $\mathrm{Ca}^{2+}$ sensor that requires sufficient $\mathrm{Ca}^{2+}$ concentrations. ${ }^{18}$ Drugs that induce an increased level of calcium might act as an activator of PKC. Since vitamin D is crucial in the calcium absorption process, we adopt the calcium tablet add on vitamin D therapy. Other studies also suggest that supplementation of sufficient $(1000 \mathrm{mg})$ elemental calcium alone did not show antidepressant effects in patients with depression ${ }^{22}$, while patients who received $500 \mathrm{mg}$ of calcium daily plus vitamin D (20000 IU or $40000 \mathrm{IU} /$ week) experienced antidepressant effects. ${ }^{23}$ Vitamin D showed potential antidepressant effects and alleviated inflammation, as suggested in peripheral blood markers. ${ }^{23}$ Vitamin D supplementation could also downregulate the gene expression of the inflammatory cytokines TGF- $\beta$ and nuclear factor kappa B (NF- $\mathrm{KB})$ and regulate the gene expression of PKC in PBMC, as evidenced by a randomised double-blind placebo-controlled trial. ${ }^{24}$ Thus, based on the vitamin $\mathrm{D}$ and calcium tablet evidence and previous research, we planned to give our patients vitamin D at $800 \mathrm{IU}$ daily plus calcium at $500 \mathrm{mg} / \mathrm{d}$ as an add-on with escitalopram in our study.

We hypothesised that PKC activation might reduce M1 microglia activation and release proinflammatory cytokines such as TNF- $\alpha$, finally alleviating depressive symptoms in TRD. As such, we speculate that TNF- $\alpha$ blocker will have a potential antidepressant effect. Many studies have been published about the antidepressant effect of TNF- $\alpha$ infliximab. ${ }^{65}$ Infliximab is a type of humanmouse monoclonal antibody ${ }^{26}$ and its method of delivery is via intravenous injection. In the current study, we chose another TNF- $\alpha$ blocker, golimumab, a human monoclonal antibody, ${ }^{27}$ since it can be delivered subcutaneously even at home. ${ }^{27}$ Hopefully, golimumab might improve safety and patient adherence compared with infliximab in patients with TRD.

In our study, in accordance with the current clinical perspective, research on cadavers and using a chronic mild stress (CMS) animal model of depression is performed concurrently to explore the upper and downstream of PKC signal pathway. Current evidence suggests that PKC might be regulated by the activation of chemokine-like receptors, including CX3CL1/ CX3CR1, which might also contribute to the microglial activation and pathogenesis of depression. ${ }^{28}$ When PKC is activated, it can regulate microglial polarisation via a downstream signalling pathway. Evidence has suggested that microglial polarisation to the M2 phenotype and a reduction in oxidative stress are mediated through
AMP-activated protein kinase (AMPK), mammalian target of rapamycin (mTORC) and nuclear factor erythroid 2-related factor 2 (Nrf2) activation. ${ }^{29}$ In our project, we will evaluate the upper and downstream of signalling pathways of PKC using single cell sequencing, qPCR, ELISA, western blotting and CRISPR genomic editing method in cadavers and the CMS animal model of depression.

There are some limitations of this design. First, we chose to administer calcium tablet $500 \mathrm{mg} / \mathrm{d}$ and Vitamin D $800 \mathrm{IU} / \mathrm{d}$ add on escitalopram. We chose the above doses based on the results of earlier studies and drug instructions $^{30}$, but a higher dose might be more effective. Second, we did not balance the sample size in terms of male and female participants and the inflammation levels across groups. However, we plan to adopt the pretreatment values (of CRP and Vitamin D) as covariates to correct the possible differences in baseline values during the data analysis process. Based on the current study, we hope to clarify the PKC-mediated microglial polarization pathway in the pathogenesis of depression and develop new strategies for treating TRD.

\section{Author affiliations}

${ }^{1}$ Department of Psychiatry, Shanghai Mental Health Center, Shanghai Jiao Tong University School of Medicine, Shanghai, China

${ }^{2}$ Department of Psychology, Shandong Provincial Hospital affiliated to Shandong First Medical University, Jinan, Shandong, China

${ }^{3}$ Department of Neurology, Renji Hospital, Shanghai Jiao Tong University School of Medicine, Shanghai, China

${ }^{4}$ Clinical Research Center and Division of Mood Disorders, Shanghai Mental Health Center, Shanghai Jiao Tong University School of Medicine, Shanghai, China

${ }^{5}$ College of Life Sciences, Chinese Academy of Sciences, Beijing, China

${ }^{6}$ CAS Center for Excellence in Brain Science and Intelligence Technology, Chinese Academy of Science, Shanghai, China

${ }^{7}$ Shanghai Key Laboratory of Psychotic Disorders, Shanghai, China

Acknowledgements We thank Professor Aimin Bao of the Department of Neurobiology at Zhejiang University School of Medicine for her kind help in the experimental design on brain tissue from cadavers.

Contributors All the authors have read and approved the manuscript before submission. XG and RM drafted/revised the manuscript for its content, including medical writing, study concept, design, interpretation of data, acquisition of data and development of clinical algorithms. FW, LC, RZ, YW, JH, YZ, YY, GZ, ZL and JW revised the manuscript for contents, including medical writing, study concept, design, interpretation of data and acquisition of data. YF revised the manuscript for content, including medical writing and design.

Funding This work was supported by the National Key R\&D Program of China (2016YFC1307100), the National Natural Science Foundation of China (81930033, 81771465, 81201057), the Shanghai Key Project of Science \& Technology (2018SHZDZX05), the National Key Technologies R\&D Program of China (2012BAl01B04) and the Innovative Research Team of High-level Local Universities in Shanghai.

Competing interests None declared.

Patient consent for publication Not required.

Ethics approval This study has been approved by the Ethics committee of Shanghai Mental Health Center at Shanghai Jiao Tong University (Ethics approval number: 2020-01). Any modifications to the protocol will require a formal amendment to the protocol. Such amendment will be agreed upon by Institutional Review Board, IRB, Shanghai Mental Health Center.

Provenance and peer review Not commissioned; externally peer reviewed.

Data availability statement Data are available upon request. 
Open access This is an open access article distributed in accordance with the Creative Commons Attribution Non Commercial (CC BY-NC 4.0) license, which permits others to distribute, remix, adapt, build upon this work non-commercially, and license their derivative works on different terms, provided the original work is properly cited, appropriate credit is given, any changes made indicated, and the use is non-commercial. See: http://creativecommons.org/licenses/by-nc/4.0/.

\section{ORCID iDs}

Xiaoyun Guo http://orcid.org/0000-0003-2738-3868

Jun Chen http://orcid.org/0000-0002-3837-2761

Yiru Fang http://orcid.org/0000-0002-8748-9085

\section{REFERENCES}

1 Wickham S, Bentley L, Rose T, et al. Effects on mental health of a UK welfare reform, universal credit: a longitudinal controlled study. Lancet Public Health 2020;5:e157-64.

2 Duman RS, Aghajanian GK, Sanacora G, et al. Synaptic plasticity and depression: new insights from stress and rapid-acting antidepressants. Nat Med 2016;22:238-49.

3 Somani A, Kar SK. Efficacy of repetitive transcranial magnetic stimulation in treatment-resistant depression: the evidence thus far. Gen Psychiatr 2019;32:e100074.

4 Jha MK, Minhajuddin A, Gadad BS, et al. Interleukin 17 selectively predicts better outcomes with bupropion-SSRI combination: novel T cell biomarker for antidepressant medication selection. Brain Behav Immun 2017;66:103-10.

5 Ma K, Zhang H, Baloch Z. Pathogenetic and therapeutic applications of tumor necrosis factor- $\alpha$ (TNF- $\alpha$ ) in major depressive disorder: a systematic review. Int J Mol Sci 2016;17:733.

6 Bekhbat M, Chu K, Le N-A, et al. Glucose and lipid-related biomarkers and the antidepressant response to infliximab in patients with treatment-resistant depression. Psychoneuroendocrinology 2018;98:222-9.

7 Tang Y, Le W. Differential roles of M1 and M2 microglia in neurodegenerative diseases. Mol Neurobiol 2016;53:1181-94.

8 Attwells S, Setiawan E, Wilson AA, et al. Replicating predictive serum correlates of greater translocator protein distribution volume in brain. Neuropsychopharmacology 2020;45:925-31.

9 Liu Y, Zhang T, Meng D, et al. Involvement of CX3CL1/CX3CR1 in depression and cognitive impairment induced by chronic unpredictable stress and relevant underlying mechanism. Behav Brain Res 2020;381:112371.

10 Guo X, Rao Y, Mao R, et al. Common cellular and molecular mechanisms and interactions between microglial activation and aberrant neuroplasticity in depression. Neuropharmacology 2020;181:108336.

11 Zhang L, Zhang J, You Z. Switching of the microglial activation phenotype is a possible treatment for depression disorder. Front Cell Neurosci 2018;12:306.

12 Hong S, Beja-Glasser VF, Nfonoyim BM, et al. Complement and microglia mediate early synapse loss in Alzheimer mouse models. Science 2016;352:712-6.

13 Shi D-D, Huang Y-H, Lai CSW, et al. Ginsenoside Rg1 prevents chemotherapy-induced cognitive impairment: associations with microglia-mediated cytokines, neuroinflammation, and neuroplasticity. Mol Neurobiol 2019;56:5626-42.

14 Strawbridge R, Hodsoll J, Powell TR, et al. Inflammatory profiles of severe treatment-resistant depression. J Affect Disord 2019;246:42-51.

15 van der Vorst EPC, Theodorou K, Wu Y, et al. High-density lipoproteins exert pro-inflammatory effects on macrophages via passive cholesterol depletion and PKC-NF-KB/STAT1-IRF1 signaling. Cell Metab 2017;25:197-207.

16 Mohanraj M, Sekar P, Liou H-H, et al. The mycobacterial adjuvant analogue TDB attenuates neuroinflammation via Mincle-independent PLC- $\gamma 1 /$ PKC/ERK signaling and microglial polarization. $\mathrm{Mol}$ Neurobiol 2019;56:1167-87.

17 Guo X, Li Z, Zhang C, et al. Down-regulation of PRKCB1 expression in Han Chinese patients with subsyndromal symptomatic depression. J Psychiatr Res 2015;69:1-6.

$18 \mathrm{Hu} \mathrm{L}$, Zhang S, Wen $\mathrm{H}$, et al. Melatonin decreases M1 polarization via attenuating mitochondrial oxidative damage depending on UCP2 pathway in prorenin-treated microglia. PLoS One 2019;14:e0212138.

19 Zhou H, Jang H, Fleischmann RM, et al. Pharmacokinetics and safety of golimumab, a fully human anti-TNF-alpha monoclonal antibody, in subjects with rheumatoid arthritis. J Clin Pharmacol 2007;47:383-96.

20 Khoraminya N, Tehrani-Doost M, Jazayeri S, et al. Therapeutic effects of vitamin $D$ as adjunctive therapy to fluoxetine in patients with major depressive disorder. Aust N Z J Psychiatry 2013;47:271-5.

21 Li X, Tian X, Lv L, et al. Microglia activation in the offspring of prenatal poly I: $C$ exposed rats: a PET imaging and immunohistochemistry study. Gen Psychiatr 2018;31:e000006.

22 Bertone-Johnson ER, Powers SI, Spangler L, et al. Vitamin D supplementation and depression in the Women's Health Initiative calcium and vitamin D trial. Am J Epidemiol 2012;176:1-13.

23 Jorde R, Sneve M, Figenschau Y, et al. Effects of vitamin D supplementation on symptoms of depression in overweight and obese subjects: randomized double blind trial. J Intern Med 2008;264:599-609.

24 Haddad Kashani H, Seyed Hosseini E, Nikzad H, et al. The effects of vitamin D supplementation on signaling pathway of inflammation and oxidative stress in diabetic hemodialysis: a randomized, doubleblind, placebo-controlled trial. Front Pharmacol 2018;9:50.

25 Şahin TD, Karson A, Balcı F, et al. Tnf-Alpha inhibition prevents cognitive decline and maintains hippocampal BDNF levels in the unpredictable chronic mild stress rat model of depression. Behav Brain Res 2015;292:233-40.

26 Derzi M, Johnson TR, Shoieb AM, et al. Nonclinical evaluation of PF06438179: a potential biosimilar to Remicade ${ }^{\circledR}$ (infliximab). Adv Ther 2016;33:1964-82.

27 Tanaka Y, Harigai M, Takeuchi T, et al. Clinical efficacy, radiographic progression, and safety through 156 weeks of therapy with subcutaneous golimumab in combination with methotrexate in Japanese patients with active rheumatoid arthritis despite prior methotrexate therapy: final results of the randomized GO-FORTH trial. Mod Rheumatol 2016;26:481-90.

28 Guo $X, F u$ Y, Xu Y, et al. Chronic mild restraint stress rats decreased CMKLR1 expression in distinct brain region. Neurosci Lett 2012;524:25-9.

29 Wang Y, Huang Y, Xu Y, et al. A dual AMPK/Nrf2 activator reduces brain inflammation after stroke by enhancing microglia $\mathrm{M} 2$ polarization. Antioxid Redox Signal 2018;28:141-63.

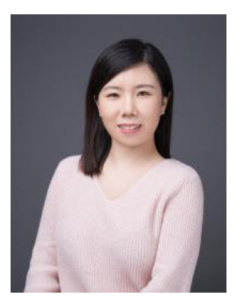

Dr. Xiaoyun Guo obtained a bachelor's degree of medicine in 2001, a master's degree in psychiatry in 2004, and a PhD in psychiatry from Fudan University, Shanghai, China in 2007. She has been working at Shanghai Mental Health Center since 2007, where she is currently an associate chief physician. She is previously an associate research scientist at Yale University School of Medicine, department of cellular and molecular physiology. Her research interest focuses on the genetic and immunological mechanism of depression. 